\title{
Active by nature: exploring cancer survivors' exercise barriers, facilitators, preferences, and psychosocial benefits of engaging in outdoor physical activity
}

\author{
Iris A. Lesser ${ }^{1}$ (D) C. P. Nienhuis ${ }^{1} \cdot$ L. Belanger ${ }^{2}$ \\ Received: 19 September 2020 / Accepted: 7 December 2020 / Published online: 6 January 2021 \\ (C) The Author(s), under exclusive licence to Springer-Verlag GmbH, DE part of Springer Nature 2021
}

\begin{abstract}
Introduction Large numbers of cancer survivors struggle with mental health after cancer diagnosis. Cancer survivors are encouraged to engage in physical activity in order to improve physical and mental health. Additional benefits to physical activity engagement in natural environments have been reported but this has not been explored in cancer survivors.

Methods Study participants had to be over the age of 19, a Canadian resident, and have had a cancer diagnosis. Recruitment to complete an online survey occurred through social media and snowball sampling. The data collected included physical activity participation, preferences and location, barriers and facilitators of engagement in outdoor physical activity, nature-related questions, and measures of psychosocial health. The sample was split by the number of outdoor physical activity minutes $(>150 \mathrm{~min}$ per week). Correlations were computed to examine the role of outdoor physical activity minutes on measured psychosocial health outcomes.

Results One hundred and fourteen $(N=114)$ cancer survivors completed the online questionnaire. More than half of the respondents indicated that an outdoor environment was central to their physical activity of choice with walking identified as the most common outdoor physical activity. Group support was the main expected facilitator of success in an outdoor walking program. Outdoor active participants were significantly more motivated and confident to be physically active and reported significantly more benefit and enjoyment in being physically active than outdoor inactive participants. Minutes of outdoor physical activity was significantly correlated with subjective happiness, nature relatedness, and higher quality of life. No significant correlations were found between minutes of outdoor physical activity and generalized anxiety.

Conclusion While future research is needed to further explore the role of nature in cancer survivor psychosocial health, we believe that our data suggests preference and benefit for outdoor physical activity in cancer survivors.
\end{abstract}

Keywords Cancer survivor $\cdot$ Physical activity $\cdot$ Mental health $\cdot$ Nature $\cdot$ Environment $\cdot$ Walking

\section{Introduction}

The number of cancer survivors is increasing due to advances in preventative screening, medical intervention, and an aging population [26]. Cancer survivorship is defined as inclusive of the entire cancer continuum, from the initial diagnosis through

Iris A. Lesser

Iris.lesser@ufv.ca

1 School of Kinesiology, University of the Fraser Valley, 45190 Caen Avenue, Chilliwack, BC, Canada

2 Knights Cabin, Knights Cabin Cancer Retreats, 1612-17th Avenue SW, Calgary T2T 0E3, Canada the lifespan. Cancer survivors may suffer from acute and chronic, physical, and psychosocial health challenges due to cancer treatment and recovery [22]. A large numbers of cancer survivors continue to struggle with mental health repercussions such as fear of cancer recurrence, post-traumatic stress, anxiety, and depression [38]. Despite the need for broad and substantive survivorship care, specifically in relation to late and long-term side effects, there is a lack of supportive programming for cancer survivors [11].

Cancer survivors are strongly encouraged to avoid inactivity and engage in at least $150 \mathrm{~min}$ of moderate to vigorous physical activity per week in order to improve physical, mental [7], social, and spiritual health [6]. Despite the known multi-faceted benefits of physical activity for cancer survivors [7], physical activity levels in cancer survivors tend to be 
lower than the general population $[12,13]$ with a reduction of one third in physical activity levels following diagnosis [2]. Although cancer survivors continue to report high rates of fatigue, $50.2 \%$ were interested in exercise and $52.5 \%$ felt able to exercise despite their reported fatigue [4]. Exercise barriers mainly related to health or environmental factors with physical activity capable of alleviating a number of the reported health barriers [4]. While physical activity appears to be beneficial for improving health outcomes in cancer survivors, it is less clear whether the physical activity environment may contribute to the positive outcomes of physical activity for mental health.

Over the past decade, an increasingly large body of literature supports the notion that time spent in nature has a positive impact on mental and psychological well-being [15, 20, 21]. It also appears that these benefits are in addition to those experienced from physical activity alone. For instance, Mayer et al. (2009) [21] found that those participating in a 15-min walk in a natural setting reported significantly more emotional wellbeing than those participating in a 15-min walk in an urban setting. Specific to anxiety, Mackay and Neill (2010) [19] found that the greenness of the environment while exercising had a greater impact on state anxiety than exercise alone. This has resulted in the coining of the term "green exercise" to describe the additional benefits of physical activity engagement in natural environments [20]. Despite these noted benefits, there is a paucity of research related to the potential benefits of the physical activity environment on psychosocial outcomes in cancer survivors, a population with generally higher levels of mental duress [38].

Cancer survivor engagement, preference, and challenges that may exist regarding outdoor physical activity participation have not been well studied. Additionally, there is a lack of literature regarding the additional benefit that physical activity environment may have on the psychosocial health of cancer survivors. A narrative review found that future research is required to further develop the emerging relationship found between nature and cancer survivors' well-being [28] Therefore, this study aims to explore the preferences, barriers, and facilitators for outdoor physical activity in cancer survivors and to examine whether engagement in outdoor physical activity impacts psychosocial outcomes.

\section{Methods}

\section{Participants}

In order to participate in the study, participants had to be over the age of 19, a Canadian resident, and have had a cancer diagnosis. Recruitment through Canadian cancer organizations, Facebook marketing, and snowball sampling directed participants to complete an online survey. While 180 cancer survivors began the online survey, 66 responses were incomplete and therefore removed from the dataset. Incomplete responses were categorized as discontinuing the survey after completion of less than half the questionnaire. A resultant participant sample of 114 adult Canadian cancer survivors was included in the study. This study received approval from the Human Research Ethics Boards at the University of the Fraser Valley, and all participants provided informed consent.

\section{Measures}

Participants completed questionnaires using an online survey software (Survey Monkey) between the Fall of 2019 and Summer of 2020. The survey comprised six parts: demographics, including cancer diagnosis and treatment, physical activity participation, physical activity environment and its determinants adapted from Lawton et al. (2017) [15], barriers and facilitators of engagement in outdoor physical activity in the cancer survivor population, nature-related questions, and validated measures of psychosocial health and well-being.

\section{Physical activity participation}

Participants reported their current physical activity levels using the Godin Leisure Questionnaire [10], which was utilized to calculate a total Godin score as well as to compute the amount of moderate and vigorous physical activity participation. Additionally, participants specified the total number of minutes in which they participated in outdoor physical activity. Further questions were asked to identify types of physical activities chosen, as well as barriers and facilitators of physical activity engagement.

\section{Physical activity environment}

Questions relating to physical activity environment included having participants identify where they engage in their chosen physical activity as well as whether the environment was central to their chosen activity type. General location was categorized based on participant responses (e.g., on trails, in the home). Further, participants indicated whether their physical activity occurred indoors, outdoors where nature is incidental to the experience (incidental meaning that the natural world was not essential to the experience), or outdoors where nature is central to the experience (central meaning where the natural environment is essential and important to the experience) [15].

\section{Nature relatedness}

Specific items from the Nature Relatedness Scale (NRS) (Nisbet et al. 2009) [25] were utilized based on their impact on physical activity behavior. The NRS asks questions about nature relatedness in the context of measuring an individuals' 
affective cognitive and physical relationship with the natural world. Each subscale uses a 5-point Likert scale ranging from 1 (disagree strongly) to 5 (agree strongly), whereby higher scores indicate a stronger connection with nature. Participants were also requested to report their connectedness to nature on a scale of 1 to 10 using a sliding scale.

\section{Anxiety}

The Generalized Anxiety Disorder (GAD-7) scale was used to assess anxiety [27]. The GAD-7 is considered an excellent screening tool for identifying people with generalized anxiety disorders [27]. The validity of GAD-7 was substantiated in a primary care sample with a sensitivity value of 0.89 and a specificity value of 0.82 [27].

\section{Fatigue}

Functional assessment of chronic illness therapy (FACITfatigue scale) was used to assess fatigue in cancer survivors [8]. The FACIT-fatigue scale is a 13-item instrument designed to assess fatigue/tiredness and its impact on daily activities and functioning in a number of chronic diseases. The questionnaire was originally used to assess cancerrelated fatigue and has shown good reliability and validity in a sample of cancer patients [37]. A higher score is indicative of a higher quality of life.

\section{Happiness}

Happiness was assessed using the subjective happiness scale (SHS). The SHS is a four-item scale of global subjective happiness in the present moment [18].

\section{Statistical analysis}

Demographics were reported as means and standard deviations. The sample was split by the number of outdoor physical activity minutes ( $>150 \mathrm{~min}$ per week), and $t$ tests were used to compare group differences. Correlations were computed to examine the role of outdoor physical activity minutes on measured psychosocial health outcomes.

\section{Results}

A participant sample of 114 cancer survivors was collected for this study. Participants ranged in the age from 25 to $82(\mathrm{M}=$ $53.6, \mathrm{SD}=14.06)$, and the majority $(76.3 \%)$ of the participants indicated that they were female. Table 1 presents additional demographic data, illustrating that the majority of the participants were married or in a domestic relationship $(60.6 \%)$, had completed university or college $(40.4 \%)$, and
Table 1 General demographic characteristics of participants

\begin{tabular}{|c|c|c|}
\hline Characteristic & $n$ & $\%$ \\
\hline \multicolumn{3}{|c|}{ Highest education level completed } \\
\hline Completed high school & 6 & 5.3 \\
\hline Some university or college & 25 & 21.9 \\
\hline Completed university/college & 46 & 40.4 \\
\hline Some graduate school & 4 & 3.5 \\
\hline Completed graduate school & 23 & 20.2 \\
\hline No response & 10 & 8.8 \\
\hline \multicolumn{3}{|l|}{ Annual household income (\$) } \\
\hline$\$ 0-20,000$ & 5 & 4.4 \\
\hline$\$ 20,000-39,999$ & 9 & 7.9 \\
\hline$\$ 40,000-59,999$ & 14 & 12.3 \\
\hline$\$ 60,000-79,999$ & 27 & 23.7 \\
\hline$\$ 80,000+$ & 43 & 37.7 \\
\hline No response & 16 & 14 \\
\hline \multicolumn{3}{|l|}{ Marital status } \\
\hline Married/domestic & 69 & 60.6 \\
\hline Widowed/divorced/separated & 22 & 19.2 \\
\hline Never married & 13 & 11.4 \\
\hline No response & 10 & 8.8 \\
\hline \multicolumn{3}{|l|}{ Current employment status } \\
\hline Full time & 36 & 31.6 \\
\hline Part time & 13 & 11.4 \\
\hline Retired & 34 & 29.8 \\
\hline Disability & 13 & 11.4 \\
\hline Unemployed & 8 & 7.0 \\
\hline No response & 10 & 8.8 \\
\hline
\end{tabular}

$N=114$

currently earned more than $\$ 80,000$ per year $(37.7 \%)$. In addition, approximately one-third of the participants worked full time (31.6\%), approximately one-third were retired (29.8\%), and the remaining one-third worked part time, were on disability, or were temporarily unemployed.

Table 2 presents cancer-related demographic information of the 114 participants. For the majority of participants (46.5\%), initial cancer diagnosis occurred more than 5 years prior to survey completion, and the stage of diagnosis varied considerably. While a majority of participants identified having breast cancer $(49.1 \%)$, a wide range of cancer types were represented, including a diverse group of "other" cancer types $(10.5 \%)$ which included cases of bladder, tonsil, salivary, and prostate and testicular cancers. Regarding treatments, a majority of participants received surgery $(78.9 \%)$, radiation therapy $(56.1 \%)$, and chemotherapy $(58.8 \%)$, and at time of survey completion, $73.7 \%$ of participants indicated that they had completed cancer treatments. Further, $82.5 \%$ of participants reported that they had been informed by their physician that they were cancer-free. 
Table 2 Cancer and health-related demographic information

\begin{tabular}{|c|c|c|}
\hline Characteristic & $n$ & $\%$ \\
\hline \multicolumn{3}{|l|}{ Cancer information } \\
\hline \multicolumn{3}{|l|}{ Time since diagnosis } \\
\hline$<1$ year & 11 & 9.6 \\
\hline $1-5$ years & 42 & 36.8 \\
\hline$>5$ years & 53 & 46.5 \\
\hline No response & 8 & 7.0 \\
\hline \multicolumn{3}{|l|}{ Stage at diagnosis } \\
\hline I & 25 & 21.9 \\
\hline II & 26 & 22.8 \\
\hline III & 25 & 21.9 \\
\hline IV & 13 & 11.4 \\
\hline Unknown & 17 & 14.9 \\
\hline No response & 8 & 7.0 \\
\hline \multicolumn{3}{|l|}{ Cancer type } \\
\hline Breast & 56 & 49.1 \\
\hline Lymphoma & 9 & 7.9 \\
\hline Colorectal & 5 & 4.4 \\
\hline Sarcoma & 5 & 4.4 \\
\hline Ovarian & 5 & 4.4 \\
\hline Thyroid & 4 & 3.5 \\
\hline Lung & 3 & 2.6 \\
\hline Skin & 2 & 1.8 \\
\hline Brain & 2 & 1.8 \\
\hline Leukemia & 2 & 1.8 \\
\hline Other & 12 & 10.5 \\
\hline No response & 9 & 7.9 \\
\hline \multicolumn{3}{|c|}{ General health information ( $\%$ affirmative) } \\
\hline High blood pressure & 21 & 18.4 \\
\hline High cholesterol & 17 & 14.9 \\
\hline Heart attack & 2 & 1.8 \\
\hline Stroke & 2 & 1.8 \\
\hline Emphysema & 1 & 0.9 \\
\hline Chronic bronchitis & 6 & 5.3 \\
\hline Diabetes & 3 & 2.6 \\
\hline Secondary cancers & 8 & 7.0 \\
\hline Angina & 1 & 0.9 \\
\hline Arthritis & 26 & 22.8 \\
\hline
\end{tabular}

Notes: $n$ and $\%$ for general health related information based on number of participants responding "yes" to the presence of conditions

In addition to cancer-specific health information, Table 2 also summarizes general health-related information as reported by participants. The most frequent health conditions reported by participants included arthritis (22.8\%), high blood pressure (18.4\%), and high cholesterol (14.9\%). As well, $52.6 \%$ of participants reported that they have never smoked, $35.1 \%$ reported that they were ex-smokers, and $2.6 \%$ are current smokers. Participants' BMI $(\mathrm{M}=26.8, \mathrm{SD}=5.56)$ was calculated utilizing self-reporting measure of height and weight. Based on participant's BMI, the majority (41.2\%) of the participants were considered a healthy weight, $30.7 \%$ were overweight, and $19.3 \%$ were obese.

Three different variables were computed to analyze participant physical activity levels. Godin Leisure Scores ranged from 0 to $126(\mathrm{M}=41.33, \mathrm{SD}=26.06)$, outdoor physical activity minutes ranged from 0 to 900 minutes $(M=206.73$, $\mathrm{SD}=180.46)$, and number of minutes of moderate-tovigorous physical activity ranged from 0 to 1260 minutes $(\mathrm{M}=232.63, \mathrm{SD}=207.12)$.

Aside from duration of physical activity, participants also reported the type and location of physical activity, as well as the impact of weather conditions on their activity levels (see Table 3 for summary). The three most commonly reported

Table 3 Physical activity behaviors and environmental characteristics

\begin{tabular}{|c|c|c|}
\hline Characteristic & $n$ & $\%$ \\
\hline \multicolumn{3}{|l|}{ Most common type of physical activity } \\
\hline Walking/hiking & 61 & 53.5 \\
\hline Weight training & 12 & 10.5 \\
\hline Biking/cycling & 9 & 7.9 \\
\hline Jogging/running & 8 & 7.0 \\
\hline Fitness classes & 6 & 5.3 \\
\hline Yoga/stretching & 4 & 3.5 \\
\hline Swimming/aqua fitness & 4 & 3.5 \\
\hline Other & 10 & 8.8 \\
\hline \multicolumn{3}{|l|}{ Most common location of physical activity } \\
\hline In the home & 13 & 11.4 \\
\hline Neighborhood & 30 & 26.3 \\
\hline Trails, parks, recreation sites & 32 & 28.1 \\
\hline General outdoors & 16 & 14.0 \\
\hline Gym/recreation center & 20 & 17.5 \\
\hline None specified & 3 & 2.6 \\
\hline \multicolumn{3}{|l|}{ Summer weather challenges } \\
\hline Heat/humidity & 50 & 43.9 \\
\hline Poor air quality & 15 & 13.2 \\
\hline Rain/storms & 40 & 35.1 \\
\hline Tornadoes/hurricanes & 7 & 7.0 \\
\hline \multicolumn{3}{|l|}{ Winter weather challenges } \\
\hline Cold temperatures & 31 & 27.2 \\
\hline Snow & 8 & 7.0 \\
\hline Ice & 55 & 48.2 \\
\hline Rain & 18 & 15.8 \\
\hline \multicolumn{3}{|l|}{ Adaptation to outdoor weather conditions } \\
\hline Skipped physical activity & 34 & 29.8 \\
\hline Waited for weather change & 21 & 18.4 \\
\hline Did physical activity indoors & 29 & 25.4 \\
\hline Did physical activity outdoors regardless of conditions & 27 & 23.7 \\
\hline
\end{tabular}

$N=114$ 
types of physical activity included walking (53.5\%), weight training (10.5\%), and biking or cycling (7.9\%). Generally, $83.3 \%$ of participants reported that location is central to their choice of physical activity, and $57.0 \%$ indicated that the outdoors was central to their physical activity experience. Trails, parks, and recreational areas $(28.1 \%)$ and the general neighborhood $(26.3 \%)$ served as the most common locations for physical activity.

Regarding environmental conditions, heat and humidity (43.9\%) and rain and storms (35.1\%) were reported as the most common weather conditions impacting summertime outdoor physical activity. Conversely, ice (48.2\%) and cold temperatures $(27.2 \%)$ were cited as most likely to change wintertime outdoor physical activity. When asked how they participate in physical activity during poor weather conditions, the majority (29.8\%) of participants simply skipped physical activity, while $25.4 \%$ moved indoors and $23.7 \%$ participated in physical activity regardless of the weather conditions.

In order to examine the impact of outdoor physical activity on various outcomes, participants were categorized as outdoor active ( $>150 \mathrm{~min}$ of outdoor physical activity per week) or outdoor inactive. Subsequently, $t$ tests were conducted to examine between group differences, as summarized in Table 4 . The only significant result found revealed that those who spent more time outdoors also indicated greater nature relatedness.

An independent sample $t$ test revealed that participants who indicated that the outdoors was central to their physical activity experience (57.0\% of participants) also reported significantly higher levels of generalized anxiety $\left(\mathrm{M}_{\text {diff }}=2.20, \mathrm{SE}\right.$ $=.883, p=0.014)$ than participants who engaged in physical activity indoors or found the outdoors to simply be incidental to their experiences. This suggests a relationship between the importance of the outdoors and generalized anxiety.

In order to explore the barriers and facilitators for outdoor physical activity in cancer survivors, independent sample ttests were conducted to examine differences between outdoor inactive and outdoor active participants (see Table 5 for summary). Differences in general barriers and facilitators, as well as outdoor specific barriers and facilitators, were included

Table 4 Outcomes based on outdoor physical activity engagement

\begin{tabular}{|c|c|c|c|c|c|c|}
\hline \multirow[t]{2}{*}{ Outcome } & \multicolumn{2}{|c|}{$\begin{array}{l}\text { Outdoor inactive } \\
(n=53)\end{array}$} & \multicolumn{2}{|c|}{$\begin{array}{l}\text { Outdoor active } \\
(n=59)\end{array}$} & \multirow[t]{2}{*}{$t$} & \multirow[t]{2}{*}{$p$} \\
\hline & M & SD & M & SD & & \\
\hline Nature relatedness & 3.62 & .58 & 4.14 & .69 & 4.13 & $<0.001$ \\
\hline Nature connection & 68.02 & 22.47 & 72.95 & 28.51 & 0.98 & 0.330 \\
\hline GAD-7 & 5.45 & 4.51 & 5.80 & 5.52 & 0.36 & 0.721 \\
\hline FACIT-fatigue & 34.91 & 10.69 & 37.76 & 10.55 & 1.38 & 0.169 \\
\hline SHS & 5.07 & 1.23 & 5.37 & 1.30 & 1.25 & 0.214 \\
\hline
\end{tabular}

in this analysis. Tests revealed that outdoor active participants were significantly more motivated and confident to be active, and reported more benefit and enjoyment in being physically active.

Participants were requested to report which factors would make participation in an outdoor walking program easier or more difficult (see Table 6). While most participates cited several factors that would facilitate or prevent program participation, responses were categorized based on participants primary response. The most common factors that make adherence to an outdoor walking program easier included peer support $(36.8 \%)$, timing and scheduling (18.4\%), and location $(16.7 \%)$. Timing and scheduling included references to work-life integration as well as set program times that would facilitate commitment and accountability. While participants frequently referred to the exposure to the natural environment as an important component of location, this factor also included proximity to home and overall accessibility, especially for those with limited mobility.

Regarding barriers to participating in outdoor walking programs, the most common factors that would make program adherence difficult included weather (24.6\%), timing and scheduling $(20.2 \%)$, health and energy levels (14.0\%), as well as the presence of other people (14.0\%). While peer support was cited as an essential component in promoting program adherence, the presence of others was also identified as a potential barrier, exampled by "poor group dynamics" such as "unfriendly" people or feeling excluded from others. Examples of timing and scheduling barriers included conflicts with work demands and poorly organized programs. Examples of problematic locations included areas of excessive city traffic or areas with limited access to natural spaces.

Bivariate analysis was conducted to explore relationships between outdoor physical activity levels and anxiety, fatigue, and subjective happiness. Minutes of outdoor physical activity was significantly and positively correlated with subjective happiness $(r=0.19, p=0.045)$, nature relatedness $(r=0.34$, $p<0.001$ ), and higher quality of life based on FACIT-fatigue scores $(r=0.23, p=0.015)$. Additionally, a significant correlation was found between nature relatedness and subjective happiness scores $(r=0.29, p=0.002)$. No significant correlations were found between minutes of outdoor physical activity and generalized anxiety.

\section{Discussion}

To our knowledge, this is the first assessment of the preferences, barriers, and facilitators for outdoor physical activity on psychosocial health in cancer survivors. More than half of our population of cancer survivors stated that an outdoor environment was central to their physical activity of choice with walking the most common outdoor physical activity. Group 
Table 5 Barriers and facilitator to outdoor physical activity

\begin{tabular}{|c|c|c|c|c|c|c|}
\hline \multirow[t]{2}{*}{ Barriers and facilitators } & \multicolumn{2}{|c|}{$\begin{array}{l}\text { Outdoor inactive } \\
(n=53)\end{array}$} & \multicolumn{2}{|c|}{$\begin{array}{l}\text { Outdoor active } \\
(n=59)\end{array}$} & \multirow[t]{2}{*}{$t$} & \multirow[t]{2}{*}{$p$} \\
\hline & M & SD & M & SD & & \\
\hline How difficult is PA? & 2.64 & 1.11 & 2.27 & 1.88 & 1.70 & 0.902 \\
\hline How motivated are you to be PA? & 3.29 & 1.16 & 4.08 & .93 & 4.00 & 0.001 \\
\hline How planned is your PA? & 2.57 & 1.26 & 2.85 & 1.30 & 1.16 & 0.248 \\
\hline How many opportunities do you have? & 3.70 & 1.17 & 3.88 & .93 & 0.92 & 0.359 \\
\hline How beneficial is PA? & 4.19 & .88 & 4.63 & .64 & 3.04 & 0.003 \\
\hline How enjoyable is PA? & 3.49 & 1.05 & 4.14 & .92 & 3.47 & 0.001 \\
\hline How confident are you to be PA? & 3.51 & 1.15 & 4.10 & .94 & 2.99 & 0.003 \\
\hline Do you have support to be active? & 3.28 & 1.29 & 3.36 & 1.40 & 0.31 & 0.758 \\
\hline
\end{tabular}

support was the main expected facilitator of success in an outdoor walking program. Outdoor active participants were significantly more motivated and confident to be physically active and reported significantly more benefit and enjoyment in being physically active than outdoor inactive participants.

Our population of cancer survivors appears to be more active than the average reports of cancer survivors with a mean well over the physical activity guidelines of $150 \mathrm{~min}$ of moderate to vigorous physical activity per week. For instance, a systematic review on cancer survivors found that the majority were insufficiently active with reports of only 17 to $47 \%$ meeting the recommended physical activity guidelines $[3,9,24]$. Similar to our population of cancer survivors, walking has previously been reported as the preferred physical activity in mixed populations of cancer survivors of various

Table 6 Outdoor walking programs facilitators and barriers

\begin{tabular}{lcr}
\hline Characteristic & \multicolumn{2}{c}{$\%$} \\
\hline What would make adherence to walking program easier? & \\
Peer support & 42 & 36.8 \\
Timing/scheduling & 21 & 18.4 \\
Level of difficulty & 6 & 5.3 \\
Location and accessibility & 19 & 16.7 \\
Weather conditions & 11 & 9.6 \\
Pet friendly & 2 & 1.8 \\
Not applicable & 12 & 10.5 \\
What would make adherence to walking program difficult? & \\
Peers/other people & 16 & 14.0 \\
Timing/scheduling & 23 & 20.2 \\
Level of difficulty & 9 & 7.9 \\
Weather & 28 & 24.6 \\
Location and accessibility & 10 & 8.8 \\
Health and energy & 16 & 14.0 \\
Not applicable & 9 & 7.9 \\
\hline
\end{tabular}

$N=114$ age groups [31,36]. Breast cancer survivors have also reported a preference for outdoor activity with walking the main physical activity choice in the outdoors [31]. An outdoor environment may provide a good distraction during physical activity, as well as be a welcomed motivator through challenging exercise [30]. Location was highly important to physical activity preference in our study with $83.3 \%$ of participants reporting that location is central to their choice of physical activity, and $57.0 \%$ indicated that the outdoors was central to their physical activity experience. A systematic review which compared indoor exercise with exercising in natural environments found greater participant enjoyment and satisfaction outdoors as well as a greater intent to adhere to exercise in the general population [32]. Similarly, we found that cancer survivors who participated in more outdoor physical activity reported greater physical activity enjoyment and benefit. We also found that a significant reduction in perceived stress in cancer survivors who participated in a bi-weekly 8week trail walking program and a significant reduction in state anxiety after each trail walk [17].

Results also demonstrated that cancer survivors who participated in more outdoor physical activity reported significantly more motivation and confidence around physical activity engagement. When asked how they participate in physical activity during poor weather conditions, the majority (29.8\%) of participants simply skipped physical activity. This is not unique to this population with adverse weather conditions being a deterrent to outdoor exercise in the general population [34]. Recommendations for outdoor physical activity in this population should therefore include education around safe outdoor participation strategies and appropriate attire may increase outdoor physical activity engagement. In relation to facilitators of outdoor physical activity, specifically walking, cancer survivors in our study stated that the most important facilitator was peer support. In a systematic review, half of the reviewed studies found a significant relationship between social support and physical activity engagement in cancer 
survivors [1]. We also found this in our trail walking program for cancer survivors where social support was stated as highly important and ensured accountability especially in an outdoor setting with potential for adverse weather conditions [17]. However, it is important to note that there was also concern over a group environment, with group physical activity being a potential barrier if there were poor group dynamics or scheduling of activity was prohibitive to engagement.

Minutes of outdoor physical activity was significantly and positively correlated with subjective happiness and higher quality of life based on FACIT-fatigue scores. One of the most reported symptoms in cancer survivors is cancer-related fatigue [35]. Our finding that outdoor physical activity is associated with cancer-related fatigue may be beneficial for advising cancer survivors on symptom reduction. Doing activities in the natural environment, as opposed to in a hospital or gym setting, may help cancer survivors strengthen ties to activities they consider normal, as opposed to cancer related [5]. In addition, outdoor physical activity alleviates the fear and risk associated with exposure in indoor environments with many cancer survivors having suppressed immune systems that expose them to more severe viral infection than the general population [14].

In our population of cancer survivors, minutes of outdoor physical activity was significantly correlated with subjective happiness and nature relatedness. As well, a significant correlation was found between nature relatedness and subjective happiness. Nature relatedness is an index of individual experience of connection to nature and may further explain the benefits of green exercise. Green space has been shown to be a protective moderator of the negative health impact of stressful life events in the general population [33]. Specifically, each additional use of a natural environment per week is associated with about a $6 \%$ lower risk of poor mental health [23]. Martyn and Brymer (2016) [20] found a significant correlation between nature relatedness and overall anxiety while also predicting lower anxiety levels. In regular exercisers, Lawton et al. (2017) [15] found that experiential connection to nature is related to some aspects of psychological well-being and lower anxiety levels and how an individual feels toward the natural world and exercising outdoors may be more important than the amount of green exercise. While no significant relationships were found between nature relatedness and anxiety in the present sample, finding a significant correlation between nature relatedness and subjective happiness affirms that connection to nature benefits psychoemotional well-being in cancer survivors.

Surprisingly, we found that cancer survivors who participated in physical activity where outdoors was central to their physical activity experience had higher anxiety than those who participated indoors or where the outdoors was incidental to their physical activity choice. It is unknown whether in our population of cancer survivors those with higher anxiety were more likely to engage in outdoor physical activity in an attempt to cope with stress. It is also possible that this may have been due to this study running through the onset of the global coronavirus pandemic which resulted in restrictions to indoor physical activity [16] and cancer survivor supportive care [29].

There are limitations to this study. This was a crosssectional analysis of cancer survivors that began prior to the onset of the coronavirus pandemic and continued through pandemic restrictions. Therefore, there are likely changes that evolved in physical activity behavior, cancer support, and psychosocial health of cancer survivors that were due to the challenges experienced with pandemic restrictions. Recruitment of cancer survivors occurred through word of mouth and cancer organizations with participants volunteering to complete the online survey which may have led to more active and educated cancer survivors completing the study.

In conclusion, cancer survivors who engaged in more outdoor physical activity were happier, had higher quality of life, and scored higher on nature relatedness. Cancer survivors prefer outdoor walking with weather being a potential barrier and group support being a potential facilitator. While future research is needed to further explore the role of nature in cancer survivor psychosocial health, we believe that our data suggests a preference for outdoor physical activity in the cancer survivor population. Given the robust benefits of nature-based physical activity in the literature, we encourage the advocacy of outdoor physical activity among cancer survivors in an attempt to increase the number of survivors meeting physical activity guidelines and subsequently experience increased happiness and quality of life. This is not limited to, but may include cancer-specific programming that extends beyond hospitals and into nature.

Authors' contributions IAL was responsible for study design, recruitment, data analysis, manuscript writing, and review. CPN was responsible for data analysis and review. LB was responsible for study design, recruitment, and review

Funding There was no funding associated with this research

Data Availability Anonymous data is available by request

\section{Compliance with ethical standards}

Conflict of interest The authors declare that they have no conflict of interest.

Ethics approval This study received approval from the Human Research Ethics Boards at the University of the Fraser Valley

Consent to participate All participants provided informed consent.

Consent for publication The authors consent to publication 
Code availability N/A.

\section{References}

1. Barber F (2012) Social support and physical activity engagement by cancer survivors. Clin J Oncol Nurs 16(3):84-98

2. Blanchard CM, Denniston MM, Baker F, Ainsworth SR, Courneya KS, Hann DM (2003) Do adults charge their lifestyle behaviours after a cancer diagnosis. Am J Health Behav 27(3):246-256

3. Blanchard CM, Courneya KS, Stein K (2008) Cancer survivors' adherence to lifestyle behavior recommendations and associations with health-related quality of life: results from the American Cancer Society's SCS-II. J Clin Oncol 26(13): 2198-2204

4. Blaney JM, Lowe-Strong A, Rankin-Watt J, Campbell A, Gravey JH (2011) Cancer survivors exercise barriers, facilitators and preferences in the context of fatigue, quality of life and physical activity participation: a questionnaire-survey. Psycho-oncology 22(1):186194

5. Blaschke S (2017) The role of nature in cancer patients' lives: a systematic review and qualitative meta-synthesis. BMC Cancer 17(1):370

6. Burke S, Wurz A, Bradshaw A, Saunders S, West MA, Brunet J (2017) Physical activity and quality of life in cancer survivors: a meta-synthesis of qualitative research. Cancers (Basel) 9(5):53

7. Campbell KL, Winters-Stone KM, Wiskemann J, May AM, Schwartz AL, Courneya KS, Zuker DS, Matthews CE, Ligibel JA, Gerber LH, Morris GS, Patel AV, Hue TF, Perna FM, Schmidtz KH (2019) Exercise guidelines for cancer survivors: consensus statement from international multidisciplinary roundtable. Med Sci Sports Exerc 51(11):2375-2390

8. Cella DF, Tulsky DS, Gray G, Sarafian B, Lloyd S, Linn E, Bonomi A, Silberman M, Yellen SB, Winicour P, Brannon J, Eckberg K, Purl S, Blendowski C, Goodman M, Barnicle M, Stewart I, McHale M, Bonomi P et al (1993) The Functional Assessment of Cancer Therapy (FACT) scale: development and validation of the general measure. J Clin Oncol 11(3):570-579

9. Courneya KS, Katzmarzyk PT, Bacon E (2008) Physical activity and obesity in Canadian cancer survivors: population-based estimates from the 2005 Canadian Community Health Survey. Cancer 112(11):2475-2482

10. Godin G (2011) The Godin-Shephard Leisure-Time Physical Activity Questionnaire. Health Fit J Can 4(1):18-22

11. Grunfeld E (2006) Looking beyond survival: how are we looking at survivorship? J Clin Oncol 24(32):5166-5169

12. Haskell WL, Lee I, Pate RR, Powell KE, Blair SN, Franklin BA, Macera CA, Heath GW, Thompson PD, Bauman A (2007) Physical activity and public health: updated recommendation for adults from the American College of Sports Medicine and the American Heart Association. Med Sci Sports Exerc 39(8):1423-1434

13. Irwin ML, McTiernan A, Ballard-Barbash R (2004) Physical activity levels among breast cancer survivors. Med Sci Sports Exerc 36(9):1484-1491

14. Kim YJ, Lee ES, Lee YS (2019) High mortality from viral pneumonia in patients with cancer. Infect Dis 51(7):1-8

15. Lawton E, Brymer E, Clough P, Denovan A (2017) The relationship between the physical activity environment, nature relatedness, anxiety, and the psychological well-being benefits of regular exercisers. Front Psychol 8:1058

16. Lesser IA, Nienhuis CP (2020) The impact of COVID-19 on physical activity behavior and well-being of Canadians. Int J Environ Res Public Health 17:3899
17. Lesser IA, Prystupa J, Belanger L, Thomson C, Nienhuis CP (2020) A mixed-methods evaluation of a group based trail walking program to reduce anxiety in cancer survivors. Appl Cancer Res 40:10. https://doi.org/10.1186/s41241-020-00094-x

18. Lyubomirsky S, Lepper HS (1999) A measure of subjective happiness: preliminary reliability and construct validation. Soc Indic Res 46:137-155

19. Mackay GJ, Neill JT (2010) The effect of "green exercise" on state anxiety and the role of exercise duration, intensity, and greenness: a quasi-experimental study. Psychol Sport Exerc 11:238-245

20. Martyn P, Brymer E (2016) The relationship between nature relatedness and anxiety. J Health Psychol 21(7):1436-1445

21. Mayer FS, Frantz CM, Bruehlman-Senecal E, Dolliver K (2009) Why Is nature beneficial?: the role of connectedness to nature. Environ Behav 41(5):607-643

22. Miller KD, Nogueira L, Mariotto AB, Rowland JH, Yabroff R, Alfano CM, Jemal A, Kramer JL, Siegel RL (2019) Cancer treatment and survivorship statistics, 2019. CA Cancer J Clin 69(5): 363-385

23. Mitchell R (2013) Is physical activity in natural environments better for mental health than physical activity in other environments. Soc Sci Med 91:130-134

24. Nayak P, Holmes HM, Nguyen HT, Elting LS (2014) Self-reported physical activity among middle-aged cancer survivors in the United States: behavioral risk factor surveillance system survey, 2009. Prev Chronic Dis 11(9):E156

25. Nisbet EKL, Zelenski JM, Murphy SA (2009) The nature relatedness scale: linking individuals' connection with nature to environmental concern and behaviour. Environ Behav 41:715-740

26. Parry C, Kent EE, Mariotto AB, Alfano CM, Rowland JH (2011) Cancer survivors: a booming population. Cancer Epidemiol Biomarkers Prev 20(10):1996-2005

27. Plummer F, Manea L, Trepel D, McMillan D (2016) Screening for anxiety disorders with the GAD-7 and the GAD-2: a systematic review and diagnostic meta-analysis. Gen Hosp Psychiatry 39:24 31

28. Ray H, Jakubec SL (2014) Nature-based experiences and health of cancer survivors. Complement Ther Clin Pract 20(4):188-192

29. Richards M, Anderson M, Carter P, Ebert BL, Mossialos E (2020) The impact of the COVID-19 pandemic on cancer care. Nat Can 1: 565-567

30. Roe JJ, Aspinall PA, Thompson CW (2017) Coping with stress in deprived urban neighborhoods: what is the role of green space according to life stage? Front Psychol 8:1760

31. Rogers LQ, Courneya KS, Verhulst D, Markwell S, McAuley E (2008) Factors associate with exercise counselling and program preferences among breast cancer survivors. J Phys Act Health 5(5):688-705

32. Thompson Coon J, Boddy K, Stein K, Whear R, Barton J, Depledge MH (2011) Does participating in physical activity in outdoor natural environments have a greater effect on physical and mental wellbeing than physical activity indoors? A systematic review. Environ Sci Technol 45(5):1761-1772

33. Van den Berg AE, Maas J, Verheil RA, Groenewegen PP (2010) Green space as a buffer between stressful life events and health. Soc Sci Med 70(8):1203-1210

34. Wagner AL, Keusch F, Yan T, Clarke PJ (2019) The impact of weather on summer and winter exercise behaviors. J Sport Health Sci 8(1):39-45

35. Wang XS, Zhao F, Fisch MJ, O'Mara AM, Cella D, Mendoza TR, Cleeland CS (2014) Prevalence and characteristics of moderate to severe fatigue: a multicenter study in cancer patients and survivors. Cancer 120(3):425-432 
36. Wong JN, McAuley E, Trinh L (2018) Physical activity programming and counseling preferences among cancer survivors: a systematic review. Int J Behav Nutr Phys Act 15(1):48

37. Yellen SB, Cella DF, Webster K, Blendowski C, Kaplan E (1997) Measuring fatigue and other anemia-related symptoms with the Functional Assessment of Cancer Therapy (FACT) measurement system. J Pain Symptom Manag 13(2):63-74. https://doi.org/10. 1016/s0885-3924(96)00274-6
38. Yi JC, Syrjala KL (2017) Anxiety and depression in cancer survivors. Med Clin N Am 101(6):1099-1113

Publisher's note Springer Nature remains neutral with regard to jurisdictional claims in published maps and institutional affiliations. 